\title{
Evaluación de la calidad sobre la aplicación móvil fiscal para cálculo de la PTU en empresas Mexicanas
}

\author{
Quality assessment on fiscal mobile application for calculating the OCT \\ in Mexican companies
}

Juan Pedro Benítez Guadarrama

Universidad Autónoma del Estado de México

jpbenitezg@uaemex.mx

Ana Luisa Ramírez Roja

Universidad Autónoma del Estado de México

alramirezr@uaemex.mx

\section{Resumen}

Uno de los tantos problemas que afectan a las empresas mexicanas que contratan personal subordinado (trabajadores) para realizar funciones específicas, es la distribución y retención del impuesto sobre las utilidades generadas en la organización; derivado de este fenómeno, la legalidad fiscal mexicana establece la obligación a los patrones a retener el Impuesto Sobre la Renta del ingreso obtenido por el trabajador sobre utilidades recibidas; a partir de esta situación, la presente investigación tiene como propósito evaluar la calidad de la aplicación móvil fiscal para eficientar la retención o subsidio al empleo otorgado aquellos trabajadores que prestan un servicio personal subordinado por los ingresos obtenidos de la utilidades generadas en un ejercicio fiscal, con la finalidad de eficientar el cumplimiento de las obligaciones contenidas en las leyes fiscales en el territorio mexicano. La investigación fue descriptiva, correlacional, no experimental, no probabilística, conformada por 120 sujetos. Se creó un instrumento conformado por 20 ítems con 
base en la Norma ISO 9126, estructurado con escala tipo Lickert, con un alfa de Cronbach 0.90. En términos de calidad, el análisis estadístico muestra las variables con mayor valor predictivo en la calidad; los resultados predicen excelentes niveles de calidad, lo lleva a inferir que las aplicaciones tecnológicas móviles pueden incorporarse en hacer más eficientes las gestiones empresariales y su relación con las autoridades fiscales en México.

Palabras clave: Calidad, ISO 9126, PTU, evaluación, aplicación.

\section{Abstract}

One of the many problems affecting Mexican companies that hire subordinate staff (employees) to perform specific functions, is the distribution and withholding tax on profits generated in the organization, resulting from this phenomenon, the Mexican tax law establishes the obligation employers to withhold income tax from the income earned by the worker on profits received, from this situation, the present investigation is to evaluate the quality of the mobile application for efficient tax withholding or employment subsidy granted those workers providing subordinated personal service income earned from the profits generated in a tax year, in order to streamline the fulfillment of the obligations contained in the tax laws in Mexico. The research was descriptive, correlational, not experimental, non-probabilistic, comprising 120 subjects. This instrument was composed of 20 items based on ISO 9126, structured Likert scale, with a Cronbach alpha of 0.90 . In terms of quality, statistical analysis shows the most predictive variable in quality, the results predict 
excellent levels of quality, it can be inferred that applications can be incorporated into mobile technology to streamline business management and its relationship with the tax authorities in Mexico.

Key words: Quality, ISO 9126, PTU, evaluation, implementation.

Fecha recepción: Enero $2013 \quad$ Fecha aceptación: Mayo 2013

\section{Introducción}

Las gestiones empresariales son actividades vitales en toda organización, vinculados a la eficiencia operativa de cada uno de los elementos que conforman el personal, contribuyen a incrementar los resultados obtenidos reflejados en las utilidades de la organización, es vital para una empresa demostrar las utilidades obtenidas al personal. La legalidad mexicana obliga a los patrones o dueños de las empresas a entregar un porcentaje y a retener el impuesto que se deriva de dicho ingreso, por lo cual, la gestión tributaria debe ser lo más ágil posible y cumplir con las expectativas de los trabajadores y de las autoridades hacendarias. Por ello, el desarrollo de esta propuesta consiste en diseñar una aplicación de interacción para la gestión tributaria, a través del uso de dispositivos móviles. Esta nueva aplicación fiscal móvil permitirá generar información sobre lo que se debe repartir a los trabajadores, así como también el impuesto retenido casi instantáneo con los deudores, para dar a conocer con anticipación el estado de sus obligaciones fiscales, al estar enterado del impuesto retenido por el patrón y sobre todo la retribución que recibirá por el desempeño de sus labores anuales. 
El objetivo general de la investigación versa sobre la evaluación de la calidad de la aplicación móvil fiscal para hacer más eficiente el proceso tributario en la retención o subsidio al empleo otorgado aquellos trabajadores que prestan un servicio personal subordinado por los ingresos obtenidos de las utilidades generadas en un ejercicio fiscal, con base en la legalidad mexicana, los patronales.

Con base en el artículo 123, apartado A, fracción IX, de la Constitución Política de los Estados Unidos Mexicanos, los trabajadores tienen derecho a participar en las utilidades que se generan en la organización, así mismo, una de las obligaciones ante las autoridades fiscales, los empresarios tiene la obligación de calcular la utilidad generada (art. 10, 16 LISR, 2012), y efectuar el reparto de acuerdo con los días trabajados y los salarios devengados durante el año, retener el impuesto generado por los ingresos obtenidos por los trabajadores (art. 113 LISR, 2012).

Los resultados muestran que el uso de la telefonía móvil en las prácticas fiscales mantienen un nivel de excelencia en la gestión tributaria, la percepción de los participantes cumple con los requerimientos legales y proporciona la información necesaria para aplicar las disposiciones tributarias contenidas en la legalidad fiscal mexicana; es una herramienta que puede ser auxiliar en el campo laboral, académico y gubernamental.

La importancia de incorporar aplicaciones móviles fiscales con calidad en la gestión tributaria y además contribuir con el objetivo del plan de desarrollo Económico de México es transcendental; así como, contar con evidencias que muestren el cumplimiento de los criterios e indicadores establecidos por las normas internacionales de calidad. Actualmente en la sociedad de la información y el conocimiento, las aplicaciones de software son un factor de éxito, por el cual las empresas utilizan herramientas más eficientes o eficientar sus gestiones de manera práctica, sin embargo, la calidad resulta ser un factor importante en las aplicaciones debido a que una de sus finalidades es mejorar y facilitar las operaciones internas 
minimizando sus costos y el tiempo (Omaña y Cárdenas, 2010). Pressman (2002) menciona que el proceso de la información establece mayores áreas de aplicación de los programas tecnológicos, la constante transformación va encaminada hacia a renovación de los sistemas de información de gestión (SIG), estas aplicaciones proporcionan datos representativos para facilitar las operaciones comerciales o gestionar la toma de decisiones; además de las actividades obligadas en el procesamiento de datos, las aplicaciones móviles promueven también la realización del cálculo interactivo. Asimismo, debido al uso generalizado y la confianza de las personas en las aplicaciones, es importante resaltar la garantía en el cumplimiento de la calidad y confiabilidad.

Moreno (2008) desarrolló un modelo para la evaluación de la calidad en uso de sitios Web, con base en el estándar ISO/IEC 9126 - 4, denominada SW-AQUA, mide cuatro aspectos: efectividad, productividad, seguridad y satisfacción en el área de control escolar del Instituto Tecnológico de Motul con una muestra de 8 participantes, el estudio demostró el nivel de calidad en su uso, determinando un nivel excelente en su eficacia, productivo, seguro y satisface las necesidades del usuarios en los niveles estándar de calidad.

Así mismo, Solarte (2009) diseñó un modelo de calidad para procesos de software, en el cual realizó un meta análisis de los modelos de calidad: la norma ISO/IEC, la integración del modelo de maduración de la capacidad CMMI y el modelo de calidad IT MARK para las PYMES, para determinar los beneficios e inconvenientes que presenta el desarrollo de software con calidad; determinando que el modelo CMMI contribuye a mejorar los procesos de trabajo y el modelo para obtener la certificación.

En un estudio realizado por Chaustre, Bolaños \& Navia (2010) en Colombia, un acercamiento a las prácticas de calidad de las aplicaciones móviles en las MiPyMES, se puede apreciar que el nivel de la calidad del software a partir de métricas de 
calidad es escasa o nula, debido a que para las empresas es importante cumplir con los requisitos planteados por el cliente y sin considerar la certificación ni evaluación de calidad, la única forma que tienen de saber si su producto es adecuado y aceptado por su cliente, ya que gran parte de los desarrolladores, se ocupa solo de crear software sin emplear algún modelo o estándar para medir la calidad de sus proyectos, ni sobre las características de calidad del producto, debido a que los procesos de desarrollo se encuentran basados en una completa especificación de requerimientos, construcción y pruebas; presentan desajustes al desarrollo rápido de aplicaciones, implicando en algunas ocasiones que se deba sacrificar la calidad del producto de software para favorecer las entregas del usuario, la rapidez y el bajo costo.

Omaña y Cárdenas (2010), en una contribución para el desarrollo de software con calidad, realizaron una estudio documental no experimental, descriptivo y transeccional a la Universidad Simón Bolívar, Venezuela; denominado Manufactura Esbelta, con miras a que las universidades que imparten carreas en el área de computación, sistemas o informática, puedan formar profesionales capaces de responder a las necesidades del entorno, partiendo de la premisa que los desarrollos de software efectuados a la fecha no satisfacen las expectativas de tiempo de desarrollo, fiabilidad, mantenibilidad, portabilidad y calidad. Se evaluó la versión 4 del SQLfi, bajo un modelo sistémico de calidad del software (MOSCA) aplicada a una población de 26 sujetos con una muestra intencional de 11, obteniendo un nivel sistémico de calidad nulo, por lo que concluyeron en la propuesta de la adopción de un modelo de desarrollo para la construcción de software de calidad basado en estándares establecidos de manufactura esbelta (Lean Manufacturing). La contribución corresponde a una mejora de la calidad sistémica de desarrollo software que permite obtener productos en forma ágil, a un costo razonable y con los recursos presupuestados. El prototipo del modelo permite identificar las 
fortalezas y debilidades de los productos de software estudiados. Al evaluar los productos de software con el prototipo, verificaron el cumplimiento de los mismos con respecto a los requerimientos críticos de calidad establecidos por el interesado en la evaluación y al mismo tiempo utilizaron los resultados para mejorarlos. Dado que la evaluación es sistémica, identifican los procesos que influyen sobre características determinadas de los productos.

Por otro lado, Santoveña (2010) diseñó un instrumento de la calidad de los cursos virtuales de la UNED en España, el instrumento consta de 36 items organizado en tres dimensiones: Calidad general del entorno y metodología, calidad técnica (navegación y diseño) y la calidad de recursos multimedia; a fin de presentar una propuesta de mejora.

Otro modelo es el propuesto por Bertoa y Villecillo (2010) para componentes de software en el que los autores adaptan la norma ISO/IEC 9126 a los componentes COTS (Commercial off-the-shelf). Rodríguez (2010) presenta una metodología para la evaluación de la calidad en los modelos UML, formada por un conjunto estructurado de procesos orientado a la evaluación de la calidad, donde a partir de una revisión de estándares, normas y metodologías relacionadas con la evaluación de la calidad del software, elaboró la metodología EVVE, bajo los siguientes principios: formada por un conjunto estructurado de procesos, orientado a la relación con el cliente y la externalización de la evaluación de la calidad, fácilmente adaptable; sin embargo identifica el qué, cuándo, y el quién, de cada una de las fases y actividades de los procesos, así como la secuencia de pasos que se debe seguir a la hora de llevar a cabo la evaluación; por lo que podemos observar que no existe a la fecha un instrumento que permita evaluar la calidad sobre una aplicación móvil fiscal específica.

La calidad es un factor de eficacia en la Ingeniería del Software, entre sus finalidades está optimizar los recursos tecnológicos en su desarrollo, esto significa un 
incremento en la productividad y competitividad organizacional; en estos tiempos las organizaciones de todo el mundo reconocen que la calidad del producto puede traducirse en ahorro de erogaciones. Las empresas desarrolladoras de software no son la excepción, motivo por el cual en tiempos actuales el crecimiento en aplicaciones tecnológicas han realizado intensos trabajos para aplicar el concepto de calidad en éste ámbito (Abud, 2010).

Los empresarios y trabajadores requieren aplicaciones móviles digitales de simplificación, con el objetivo de cumplir con las obligaciones ante las dependencias gubernamentales; la tecnología de mayor alcance por el usuario es el teléfono inteligente, el cual permite realizar diferentes acciones que cubren con sus necesidades inmediatas, en el contexto empresarial, podría ser una herramienta con propósitos de mejora en los procesos de la gestión tributaria, así como en el cálculo de las utilidades que se distribuyen internamente en las organizaciones; de manera práctica, sencilla, confiable y sobre todo oportuna; con el propósito de dar cumplimiento a las contribuciones, se diseñó una aplicación para ejecutarse en teléfono inteligente con disposiciones contenidas en la ley del Impuesto Sobre la Renta.

La creación de aplicaciones móviles requiere la constante vinculación con los modelos de calidad que permitan evidenciar los niveles de excelencia de los productos e instrumentos de medición incluyendo variables, indicadores y factores califiquen cada uno de los atributos que lo conforma. Esta investigación se creó un instrumento con base en la Norma ISO 9126 (ISO 9126, 2011), la norma 14958 en el apartado 5 (ISO 14958, 2010), establece criterios internos y externos para la evaluación de la calidad de tecnologías flexibles, en tal sentido, el instrumento de medición de la calidad está conformado por 20 ítems considerando 6 variables (funcionalidad, confiabilidad, portabilidad, eficiencia, mantenibilidad, usabilidad), estructurado con escala tipo Lickert, considerando 1 excelente; 2 bueno; 3 regular; 4 
deficiente; 5 malo, el instrumento se aplicó a 120 contadores públicos certificados activos, actualmente prestan servicios de asesoría, consultoría a empresarios dedicados a realizar actividades empresariales, además de cumplir con los criterios necesarios para su uso y aplicación en la práctica fiscal.

Al iniciar la evaluación se informó a los participantes el objetivo del estudio, evaluar la aplicación fiscal móvil, se les indico que el desarrolló del modelo tecnológico móvil se diseño con base en la legislación fiscal mexicana; el proceso de instalación de la aplicación en su teléfono inteligente, como usarlo y ejecutarlo, a fin de obtener los resultados requeridos por el empresario, trabajador y profesionistas sobre los impuesto a retener sobre las utilidades ganadas por el prestador de servicios subordinado, actualmente aplicable para el año 2012, cuya obligación con el Servicio de Administración Tributaria (SAT) es enterar y pagar los impuestos, la contribución debe ser determinada, calculada y retenida por el propio empresario para ser pagada en el periodo especificado en ley; además, se especificó el tipo de tecnología donde se puede utilizar. Así mismo, se proporcionó el archivo ejecutable por medio de bluetooth al teléfono inteligente, para utilizarlo durante la práctica e iniciar el proceso de evaluación.

En la segunda fase se describieron los conceptos que integran la aplicación fiscal, se explicaron los montos que deben ingresar para obtener los resultados requeridos el usuario interesado y por la autoridad correspondiente, todos los participantes están asociados con los conceptos que se manejan para calcular el ISR.

En la tercera fase se llevó a cabo una sesión con una duración de 50 minutos aproximadamente donde se presentó la aplicación móvil, se explicó su funcionamiento, se realizaron casos prácticos reales, proporcionado datos de trabajadores para utilizar la aplicación fiscal y de esta forma se capacito a los sujetos para usar la aplicación móvil, posteriormente, se proporciono el instrumento para iniciar su evaluación. 
En el procesamiento de datos se utilizó el paquete estadístico Statical Package for te Social Sciencies (SPSS, Versión 19.0), mediante la utilización del programa se realizó el análisis descriptivo, con el propósito de tener una visión general sobre los resultados obtenidos, se realizó un análisis a través de la aplicación de las medidas de tendencia central en las variables y finalmente a cada uno de los factores predictores de calidad; un análisis inferencial con el propósito de argumentar sobre la correlación de Pearson, con el objetivo de encontrar el grado de relación existente entre las variables de la calidad; además, la regresión lineal para predecir las variables que influyen con mayor fuerza en la calidad.

La muestra fue elegida de una población de 120 sujetos, donde el $100 \%$ ( $n=120$ ) aceptaron voluntariamente participar en el estudio. El 60\% $(n=72)$ eran hombres y el $40 \%(n=48)$ mujeres, quienes emplearon la aplicación fiscal en su celular como herramienta para el cálculo de la retención o subsidio otorgado a las trabajadores (Cuadro 1).

\begin{tabular}{lcc}
\hline Género & Frecuencia & Porcentaje \\
\hline Femenino & 48 & 40 \\
Masculino & 72 & 60 \\
Total & 120 & 100.0
\end{tabular}

Cuadro 1. Tabla de distribución de la muestra por género.

Por lo que respecta a la edad de los participantes, el $31.7 \%(n=38)$ tenían 36 años, el 24.2\% ( $n=29) 37$ años, el $15.8 \%(n=19) 38$ años, el 15.8\% $(n=19)$ tenían 39 años y el $121.7 \%(n=5)$ tenían de 35 años. (Cuadro 2 ). 


\begin{tabular}{lcl}
\hline Edad & Frecuencia & Porcentaje \\
\hline 35 años & 15 & $12.5 \%$ \\
36 años & 38 & $31.7 \%$ \\
37 años & 29 & $24.2 \%$ \\
38 años & 19 & $15.8 \%$ \\
39 años & 19 & $15.8 \%$ \\
Total & 120 & $100.0 \%$
\end{tabular}

Cuadro 2. Tabla de distribución de la muestra por edad.

Del total de la muestra, los participantes usaron celulares distintos: donde predomino el $35 \%(n=42)$ que usaron Nokia, el $25 \%(n=30) L G$, el $25 \%(n=30)$ Motorola, y el tan solo el $15 \%(n=18)$ con Sony Ericsson (Cuadro 3).

\begin{tabular}{lll}
\hline Marca de celular & Frecuencia & Porcentaje \\
\hline Nokia & 42 & $35 \%$ \\
LG & 30 & $25 \%$ \\
Motorola & 30 & $25 \%$ \\
Sony Ericsson & 18 & $15 \%$ \\
Total & 120 & $100 \%$
\end{tabular}

Cuadro 3. Tabla de distribución por marca de celular.

Respecto al tipo de actividad que los participantes prestan servicios, el $60 \%$ ( $n=72)$ eran destinados al comercio, el $15 \%(n=18)$ eran destinados a servicios y el $25 \%$ 
$(n=30)$ eran destinados a la transformación e industria (Cuadro 4).

\begin{tabular}{lcc}
\hline Actividad & Frecuencia & Porcentaje \\
\hline Comercio & 72 & $60 \%$ \\
Servicios & 18 & $15 \%$ \\
Transformación & 30 & $25 \%$ \\
Total & 120 & $100.0 \%$
\end{tabular}

Cuadro 4. Tabla de distribución por actividad.

Los criterios de evaluación descriptivos: Media $(X \overline{)}$ y Desviación Estándar (DE) de los indicadores de la calidad, tenemos que el nivel de calidad global (1.085), en cada una de sus subescalas: eficiencia, portabilidad, funcionalidad, usabilidad, fiabilidad y mantenibilidad, tienden a ser excelentes (Cuadro 5).

\section{Factores}

\begin{tabular}{llllllll}
\hline $\begin{array}{l}\text { Funcionalid } \\
\text { ad }\end{array}$ & $\begin{array}{l}\text { Fiabilid } \\
\text { ad }\end{array}$ & $\begin{array}{l}\text { Usabilid } \\
\text { ad }\end{array}$ & $\begin{array}{l}\text { Eficienc } \\
\text { ia }\end{array}$ & $\begin{array}{l}\text { Mantenibilid } \\
\text { ad }\end{array}$ & $\begin{array}{l}\text { Portabilid } \\
\text { ad }\end{array}$ & $\begin{array}{l}\text { Total } \\
\text { Calida } \\
\text { d }\end{array}$ \\
1.075 & 1.025 & 1.035 & 1.025 & 1.025 & 1.035 & 1.085 \\
.101 & .095 & .095 & .085 & .085 & .085 & .075
\end{tabular}

Cuadro 5. Tabla de factores predictores de la calidad. 
Con respecto de la correlación entre subescalas, así como de la calidad, observamos que la funcionalidad correlaciona de manera positiva muy fuerte con portabilidad y eficiencia; al igual que la fiabilidad con la eficiencia, la usabilidad con mantenibilidad; la eficiencia y mantenibilidad, la mantenibilidad y la portabilidad; las demás variables se relaciona de forma positiva considerable; podemos observar que en todas la variables existe una correlación positiva muy fuerte con la calidad (Cuadro 6).

\begin{tabular}{|c|c|c|c|c|c|c|c|}
\hline & \multicolumn{7}{|c|}{ Variables de calidad } \\
\hline & $\begin{array}{l}\text { Funcionali } \\
\text { dad }\end{array}$ & $\begin{array}{l}\text { Fiabili } \\
\text { dad }\end{array}$ & $\begin{array}{l}\text { Usabili } \\
\text { dad }\end{array}$ & $\begin{array}{l}\text { Eficien } \\
\text { cia }\end{array}$ & $\begin{array}{l}\text { Mantenibil } \\
\text { idad }\end{array}$ & $\begin{array}{l}\text { Portabili } \\
\text { dad }\end{array}$ & $\begin{array}{l}\text { Calid } \\
\text { ad }\end{array}$ \\
\hline $\begin{array}{l}\text { Funcionali } \\
\text { dad }\end{array}$ & 1.0 & & & & & & \\
\hline Fiabilidad & $.715^{* *}$ & 1.0 & & & & & \\
\hline Usabilidad & $.813^{* *}$ & $.886 * *$ & 1.0 & & & & \\
\hline Eficiencia & $.914^{* *}$ & $.965^{* *}$ & $.865^{* *}$ & 1.0 & & & \\
\hline $\begin{array}{l}\text { Mantenibil } \\
\text { idad }\end{array}$ & $.875^{* *}$ & $.820 * *$ & $.966 * *$ & $.918^{* *}$ & 1.0 & & \\
\hline $\begin{array}{l}\text { Portabilida } \\
\text { d }\end{array}$ & $.928 * *$ & $.806^{* *}$ & $.855^{* *}$ & $\begin{array}{l}.869 \\
* *\end{array}$ & $.955^{* *}$ & 1.0 & \\
\hline Calidad & $.900 * *$ & $.965^{* *}$ & $.964^{* *}$ & $.953 * *$ & $.955^{* *}$ & $.929 * *$ & 1.0 \\
\hline
\end{tabular}


Discusión

La muestra estuvo compuesta por un $40 \%$ de mujeres y $60 \%$ de hombres, por lo cual establece en esta investigación mantiene una participación activa en las actividades profesionales por hombres. Las edades oscilaron entre los 35 a los 39 años, resaltado que el dato más representativo pertenece a 37 años de edad, representando el $24.2 \%$ de la muestra, lo que nos indica que este tipo de dispositivos móviles es usado por adultos jóvenes dedicados de manera constante a la gestión empresarial.

En el estudio predominó el uso de celular de marca Nokia con el 35\% con una frecuencia de 42 sujetos, contra el 25 \% restante para las marcas: LG, Motorola, con una frecuencia de 30 sujetos respectivamente; el 15\% con una frecuencia de 18 sujetos, respectivamente, resaltando que la marca predominante en el uso entre los participantes es Nokia.

Así mismo, el 100\% de los participantes conocen la determinación y cálculo del impuestos sobre la renta en la participación de los trabajadores en la utilidades, el $60 \%$ de los participantes prestan servicios en la actividad comercial, el $15 \%$ a la actividad servicios y el $25 \%$ al sector industrial, resaltando que en la zona de los participantes existe un predominio de la actividad comercial, es decir a la compra y ventas de productos.

Al conocer la determinación y cálculo del impuesto, la aplicación móvil tiene mayor aceptación por los participantes, además los conceptos incluidos son con base en las disposiciones aplicables al ejercicio 2012, así como, el salario mínimo general y la tablas emitidas por la autoridad para realizar el cálculo.

El instrumento de medición obtuvo un Coeficiente Alfa de Cronbach de $\propto=0.918$, lo que indica un nivel de confiabilidad muy bueno. La percepción de los sujetos en 
general muestra un nivel de excelencia y alto cumplimiento con respecto de la eficiencia $(X=1.025$ con desviación estándar de .085 ), portabilidad $(X=1.035$ con desviación estándar de .085), funcionalidad ( $X=1.075$ y una desviación estándar de .095), usabilidad ( $X=1.035$ con desviación estándar de .095), fiabilidad ( $X=1.025$ con desviación estándar de .095) y la mantenibilidad de la herramienta $(X=1.025$ con desviación estándar de .095); en términos generales de calidad, se obtuvo una media de 1.085 con una desviación estándar de .075 , lo que significa que la aplicación móvil presenta un excelente nivel en la calidad para ser adoptada en la práctica fiscal.

En la correlación de Pearson se encontraron veintiuna correlaciones significativas de un total de veintiún posibles. Las correlaciones más altas se dieron entre la mantenibilidad y usabilidad ( $r=.966)$, La calidad muestra correlaciones positivas significativas con la usabilidad $(r=.964)$, portabilidad $(r=.929)$, eficiencia $(r=.953)$, mantenibilidad ( $r=.955)$ y fiabilidad $(r=.965)$, funcionalidad ( $r=.900)$; esto representa que la estructura, ejecuciones y funciones contenidas en la aplicación móvil fiscal reducen el tiempo de respuesta y hacen más eficiente y eficaz uso del teléfono inteligente en los procesos de gestión tributaria.

Los coeficientes de determinación $\left(r^{2}\right)$ permitieron conocer el nivel en que cada variable independiente predice el comportamiento de la dependiente. Todas las variables mostraron alto nivel en la predicción del comportamiento de la variable calidad es la fiabilidad $\left(r^{2}=.965\right)$, la portabilidad $\left(r^{2}=.929\right)$, la eficiencia $\left(r^{2}=953\right)$ y funcionalidad $\left(r^{2}=.900\right)$; la mantenibilidad $\left(r^{2}=.955\right)$ y la usabilidad $\left(r^{2}=.964\right)$, el reconocimiento de la calidad de la aplicación permite considerar que puede ser usada por el profesionista o empresario en el cumplimiento de las obligaciones fiscales. 


\section{Conclusión}

La aplicación móvil fiscal presenta resultados significativos de calidad para el quehacer profesional, empresarial o gubernamental en las práctica fiscal, actualmente el profesionista buscan tecnologías con respuestas inmediatas para proporcionar servicios más eficientes y eficaces; así mismo, los empresarios buscan tecnologías que agilicen los procesos administrativos, financieros y gubernamentales; y las autoridades hacendarias buscan incorporar tecnologías al alcance de los empresarios y profesionistas encaminados a cumplir de forma sencilla, práctica y confiable el pago de las contribuciones.

La aplicación móvil fiscal al ser considerada con un nivel de calidad excelente por el usuario, cumple con los criterios exigibles por la norma ISO 9126 con el objetivo de mejorar los procedimientos y métodos administrativos orientados al cumplimiento de los ordenamientos fiscales a los que está sujeto el empresario o contribuyente periodo a periodo, al presentar niveles de excelencia en sus funciones específicas, no requiere de invertir gran esfuerzo ya que su diseño orienta al usuario en la inserción de datos con facilidad, sus atributos permiten de la aplicación incrementar el uso de los dispositivos portátiles de fácil ejecución, aportando solución inmediata en las prácticas fiscales, los niveles altos de eficiencia con base en el tiempo de respuesta inmediata y la utilización del tipo de recurso permiten obtener resultados inmediatos, reales y verídicos, los niveles óptimos de mantenibilidad hacen de la herramienta la permanencia en la ejecución en el momento de ser requerida y ejecutada con resultados satisfactorias; su portabilidad permite ser transferida de un lugar a otro; el excelente nivel de confiabilidad proporciona elementos suficientes para ser considerada como una herramienta fiscal con inclusión en la 
política fiscal y facilidad en las gestiones del país.

Al desarrollar tecnologías que justifiquen la calidad en los procesos fiscales caso específico la determinación y cálculo de la participación de trabajadores en la utilidades, motivo de esta investigación e incorporar tecnología móvil al área gubernamental, empresarial y profesional, ofrece importantes beneficios sociales, económicos que inciden en la gestión administrativa como la disponibilidad y obtención de información en segundos desde cualquier lugar y momento, otorgándole eficacia en la captura de datos de manera fácil y específica, brindando movilidad en la funciones y jerarquía en las organizaciones sociales, con la misión de facilitar la toma de decisiones; soluciones que se integran perfectamente a las exigencias del mundo tecnológico globalizado con la aplicaciones sencillas para la realización de una gestión fiscal eficiente y compatible con el sistema de información con el que cuente la empresa en beneficio de los sujetos de la relación tributaria mejorando los procesos gubernamentales.

Las conclusiones de este estudio revelan que las aplicaciones móviles revolucionará los procesos organizaciones y aumentará la competitividad y productividad; como resultado de la implementación de las aplicaciones móviles fomentara la eficacia tanto interna como externamente con información oportuna en el pagos de la contribuciones a pegadas a las disposiciones ejecutivas internas del país. Al suministrar aplicaciones móviles que permitan integrar el sistema tributario local (cálculo de los impuestos federales) en cualquier régimen fiscal a los que se enfrentan durante el ejercicio fiscal, se brindarán mejores oportunidades en la aceptación del gastos público, los trabajos futuros sobre esta línea de investigación pretenden incorporar este tipo de tecnología flexible en la trasferencia electrónica de datos a través del teléfono inteligente o smartphone permitiendo obtener información suficiente para verificar y comprobar la forma de determinación, calculo y pago del impuesto por este medio electrónico, tan ocupado por los usuarios en 
México a fin de evitar desplazamiento, pérdida de tiempo y uso territorial ilimitado.

\section{Bibliografía}

Bolaños \& Navia. (2010). Practicas para Ingeniería del Software. Madrid: Prenticehall.

Omaña, M. \& Cadenas, J. (2010). Manufactura Esbelta: una contribución para el desarrollo de software con calidad. Enl@ce Revista Venezolana de Información, Tecnología y Conocimiento, 7 (3), 11-26.

Prieto, M. (2005). Desarrollo de juegos con J2ME Java 2 Micro Edición. México: alfa omega.

Rodríguez, M.; Verdugo, J.; Coloma, R.; Genero, M. \& Piattini, M. (2010). Metodología para la evaluación de la calidad en los modelos UML. REICIS Revista Española de Innovación, Calidad e Ingeniería del Software, 6 (1), 16-35.

Solarte, G.; Muñoz, L.; Arias, B. (2009). Modelos de calidad para procesos de software. Scientia Et Technica, 15 (42), 375-379. 